\title{
Blasenkarzinomrisiko bei Rauchern gestiegen
}

\begin{abstract}
Dass Rauchen das Blasenkrebsrisiko erhöht, ist gut dokumentiert. Unklar ist, wie sich hier veränderte Rauchpraktiken auswirken. Eine US-Studie zeigt, dass das Blasenkrebsrisiko in jüngster Zeit stark angestiegen ist.
\end{abstract}

- US-Forscher prüften das Blasenkarzinomrisiko in Relation zu den Rauchpraktiken anhand der Interviewdaten einer großen Fall-Kontroll-Studie, die von 2001 bis 2004 in Maine, New Hampshire und Vermont $(n=1170$ urotheliale Karzinompatienten und 1413 Kontrollpatienten) durchgeführt wurde. Die Veränderungen der Blasenkarzinomrisiken wurden aus dem Vergleich der Daten der letzten NewHampshire-Studie (305 Karzinompatienten und 305 Kontrollpersonen) ermittelt. Zusätzlich wurden Daten von zwei Fall-Kontroll-Studien in New Hampshire aus den Jahren 1994-1998 und 1998-2001 (843 Fallpatienten und 1183 Kontrollpersonen) verwendet. Die Raucher - ehemalige und gegenwärtige - hatten ein bis zu fünfmal höheres Blasenkarzinomrisiko als die Nichtraucher. Ältere Studien hatten lediglich ein dreifaches Risiko ergeben.

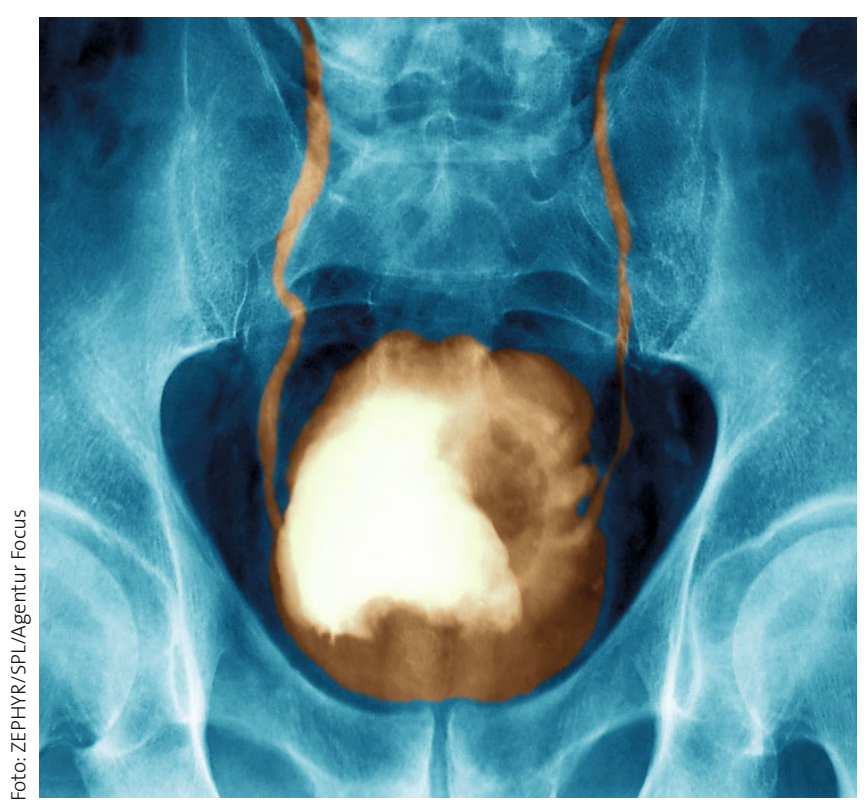

4 Eines der Risiken des Rauchens: Blasenkarzinom.
KOMMENTAR: Seit Mitte der 199oer-Jahre haben in New Hampshire die durch das Rauchen bedingten, gegenüber Nichtrauchern erhöhten Risiken für ein Blasenkarzinom vom Dreifachen auf das Fünffache zugenommen. Als Ursachen werden eine Zunahme von Blasenkrebskarzinogenen im Zigarettenrauch - durch Zusatzstoffe im Tabak - und eine intensivere Inhalation des Rauches von den heute üblichen
Zigaretten mit niedrigerem Teer- und Nikotingehalt diskutiert.

K. MALBERG =

- D. Baris et al.

A case-control study of smoking and bladder cancer risk: emergent patterns over time. Journal of the National Cancer Institute 2009;101:1553-1561; doi:10.1093/jnci/djp361

\section{Kolontherapie: eine evidenzfreie Zone}

Nicht weniger als ein längeres und gesünderes Leben versprechen die Anbieter der Kolontherapie. Die Darmreinigung soll den Körper von Allergenen befreien und Asthma, Arthrosen, Erschöpfungszustände sowie Übergewicht beseitigen. Nur leere Versprechungen oder ist an der Methode etwas dran?

- Amerikanische Forscher haben sich die Mühe gemacht zu prüfen, auf welcher evidenziellen Basis die Kolontherapie steht. Nach ausgedehnten Literaturrecherchen fanden sie 17 Publikationen mit klinischen Daten.
Die allermeisten davon waren Fallberichte oder Fallserien. Es fand sich keine einzige kontrollierte klinische Studie, die die Wirksamkeit dieser Behandlungsform kritisch überprüfte. Die Autoren schließen daraus, dass diese Therapie nicht empfohlen werden kann.

KOMMENTAR: Die Kolontherapie ist auch bei uns äußerst beliebt. Im Prinzip handelt es sich um einen hohen Einlauf mit Wasser, dem gelegentlich Kaffee, Heilkräuter oder andere Inhaltsstoffe zugesetzt werden. Meist wird die Methode eingesetzt, um das allgemeine Wohlbefinden zu fördern oder den Körper zu „entgiften”. Für den Patienten ist das Resultat, nämlich der entleerte Einlauf plus Darminhalt, häufig sichtbar plausibel. Man könnte das Ganze einfach als Unsinn abtun, wenn nicht die unübersehbare Gefahr von Komplikationen bestünde; u.a. sind Darmperforationen und kritische Elektrolytverluste beschrieben worden. Fazit: Hände weg von der Kolontherapie!

E. ERNST =

\footnotetext{
- R. D. Acosta, B. D. Cash Clinical effects of colonic cleansing for general health promotion: a systematic review. Am. J. Gastroenterol. 104 (2009) 2830-2836
} 